\title{
Preface to Special Issue on Celebrating the 100th Anniversary of Nankai University
}

The day of October 17, 2019 is the 100th birthday of Nankai University. In order to celebrate the grand 100th anniversary, we organized this special issue of Chinese Journal of Catalysis to present some recent advances in the discipline of catalysis at Nankai University.

In 1978, Professor Hexuan Li, a Chinese prestigious physical chemist, founded the Laboratory of Catalysis and Kinetics, attached in Department of Chemistry, Nankai University, where the researches were focused on zeolite synthesis and catalysis. One of most important achievements is "Template-free synthesis of zeolite ZSM-5", led by the group of Prof. Hexuan Li and Prof. Shouhe Xiang in the 1980s. The synthesis route has been applied in the industrial production of ZSM-5 zeolites from then and until now, and various zeolite catalysts have been developed. In 1997, the Institute of New Catalytic Materials Science, Nankai University, was established. Since then, the catalysis researches in Nankai University are experiencing a fast development on both fundamental theory and industrial applications. Nowadays, the research interest has been extended from traditional adsorption and catalysis to some emerging fields like photocatalysis and electrocatalysis.

As the guest editors of this thematic issue in Chinese Journal of Catalysis, we aim to present an overview of the catalysis researches in Nankai University through selected papers in the issue, which are contributed by both the faculty and alumni in Nankai University. This special issue contains 14 invited papers that cover the most important and promising aspects of catalysis and catalytic materials, including catalyst preparation technology such as atomic layer deposition and magnetron sputtering, construction of active sites in zeolites and related catalysis, ordered mesoporous carbon materials as metal-free heterogeneous catalysts, three-dimensional macroporous perovskite materials for environmental catalysis, metal-organic framework materials for photocatalytic hydrogen production, electrocatalytic active counter electrode materials for dye-sensitized solar cells, bifunctional electrocatalysts for rechargeable Zn-air batteries, self-supporting electrocatalysts for overall water splitting, and state-of-the-art catalysts for direct dehydrogenation of propane to propylene.

Finally, we would like to express our sincere gratitude to all the contributors, including the authors and reviewers, as well as the editorial staff of Chinese Journal of Catalysis for their great help and supports to make the success of this special issue.

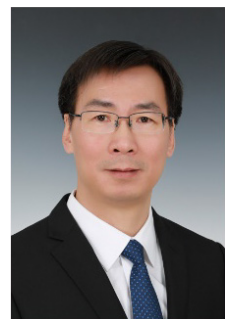

Prof. Zhong-Yong Yuan School of Materials Science and Engineering, Nankai University, Tianjin 300350, China E-mail: zyyuan@nankai.edu.cn

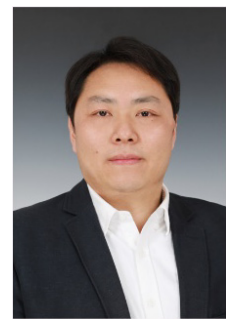

Prof. Landong Li

School of Materials Science and Engineering, Nankai University, Tianjin 300350, China

E-mail: lild@nankai.edu.cn

DOI: S1872-2067(19)63425-X| http://www.sciencedirect.com/science/journal/18722067 | Chin. J. Catal., Vol. 40, No. 9, September 2019 (C) 2019, Dalian Institute of Chemical Physics, Chinese Academy of Sciences. Published by Elsevier B.V. All rights reserved. 